\title{
Three Term Weighted Type Fractional Fourier Transform Based Generalized Hybrid Carrier and Its Application into PAPR Suppression
}

\author{
Yong LI ${ }^{\mathrm{a}, \mathrm{b}, 1}$, Zhiqun SONG ${ }^{\mathrm{a}, \mathrm{b}}$, Teng SUN ${ }^{\mathrm{b}}$, and Bin WANG ${ }^{\mathrm{a}, \mathrm{b}}$ \\ ${ }^{a}$ Science and Technology on Communication Networks Laboratory, China \\ ${ }^{\mathrm{b}}$ The 54th Research Institute of China Electronics Technology Group \\ Corporation, China
}

\begin{abstract}
To suppress the peak to average power ratio (PAPR) of wireless communication based upon multi-carrier system. We, in this paper, proposed the three term weighted type fractional Fourier transform (3-WFRFT) based generalized hybrid carrier (GHC) system. We first provide the definition of 3-WFRFT. Moreover, some useful properties of 3-WFRFT have been presented, in this paper, which will helpful to comprehend the novel 3-WFRFT transform. Furthermore, we take PAPR of the proposed algorithm, in comparison with orthogonal frequency division multiplexing (OFDM) system and single carrier modulation (SC) system under typical complementary cumulative density function (CCDF) level. It would be demonstrated that, from some numerical simulations, the proposed 3-WFRFT based GHC performs better than OFDM system and will be useful to reduce the PAPR level.
\end{abstract}

Keywords. Three term weighted type fractional Fourier transform (3-WFRFT), peak to average power ratio (PAPR), generalized hybrid carrier (GHC))

\section{Introduction}

Recently, the multi-carrier system seems popularity over wireless communications, such as digital video broadcasting (DVB) and digital audio broadcasting (DAB), wireless local area networks (WLAN) [1-4] and the $5^{\text {th }}$ generation mobile communication technology (5G) and so on. Moreover, given the less complexity and high spectral efficiency, the multiple carrier system has been some standard for most of wireless communications. However, due to the signal envelope and high fluctuation of multi-carrier, the peak to average power ratio (PAPR) is high, which will influence the application over wireless communication. Specially, when the non-linear power amplifier (PA) has been used on wireless communication, the high PAPR will cause obliviously degradation in performance.

\footnotetext{
${ }^{1}$ Corresponding Author: Science and Technology on Communication Networks Laboratory; The 54th Research Institute of China Electronic Technology Group Corporation, Shijiazhuang, Hebei,050081,China,Email:young_li_54@126.com
} 
There are many studies about PAPR suppression. In [5], the author proposed the Hadamard recursive carrier interferometry (HRCI) codes and diagonal recursive carrier interferometry (DRCI) codes to suppress the PAPR under OFDM systems. Jiang in [6] provided the Iterative Compensation algorithm to PAPR suppression. In order to reduce the complexity, Cheng in [7] proposed C-DSLM algorithm. It can be observed, from the studies recently [5-7], the communication scheme is still the DFT based OFDM system. However, the PAPR of DFT for wireless signals will be prohibitively high . We need a technology, which can both inherit the superior of OFDM and suppress PAPR, to improve the performance of wireless communications.

To this end, we in this paper, proposed the three term weighted type fractional Fourier (3-WFRFT) based generalized hybrid carrier (GHC) system to mitigate PAPR of wireless communications. The 3-WFRFT based GHC system, coupling with the components of single carrier modulation (SC) and orthogonal frequency division multiplexing (OFDM) signals. It is expected the PAPR level is lower than OFDM signals. It is demonstrated via numerical simulations, that 3-WFRFT based GHC system performs better than OFDM systems under the same channel conditions.

The rest of this paper is listed as follows. We first present The background theory to derive the 3-WFRFT and its properties in Section 2. The GHC based upon 3-WFRFT, will be given in Section 3. Furthermore some simulations based upon PAPR and discussions have been considered in Section 4. Finally, the conclusion of whole paper has been shown in Section 5.

\section{The Background Theory}

\subsection{3-Weighted Type Fractional Fourier Transform (3-WFRFT)}

There are many various forms for Multi-Weighted type Fractional Fourier Transform (M-WFRFT) according to [8-13]. However, the term $M$ can only be $4 N, N \in \mathbb{Z}$ or $M>4, M \in \mathbb{Z}$ and so on. To this end, we will proposed the 3-WFRFT.

In this section, we will provide the concept of 3-WFRFT, which is based upon 4WFRFT. In additional, M-WFRFT ( $M>4$ ) proposed in [14], is also based upon 4WFRFT. However, the relationship between M-WFRFT $(M>4)$ and 4-WFRFT has been derived in [14], but not that of 3-WFRFT and 4-WFRFT. We, in this paper, will provide the concept of 3-WFRFT and some properties, which maybe helpful to solve the problem.

The symbol of $N$-length $\mathbf{S}^{T}=\left\{s_{1}, s_{2}, \ldots, s_{N-1}, s_{N}\right\}$, and its $\alpha$-order $(\alpha \in[0,1])$ 3-WFRFT can be defined as:

$$
\mathbf{X}=\mathfrak{F}_{3}^{\alpha}[\mathbf{S}]=\mathbf{W}_{3}^{\alpha} \mathbf{S}^{T}
$$

where $\mathbf{W}_{3}$ is called the $N \times N$ 3-WFRFT matrix, and can be expressed as:

$$
\mathbf{W}_{3}^{\alpha}=\sum_{l=0}^{2} B_{l}(\alpha) \mathbf{W}_{4}^{\frac{4}{3} l}
$$

$\mathbf{W}_{4}^{\frac{4}{3} l}(l \in \mathbb{Z}, l \in[0,2])$ is the $N \times N$ 4-WFRFT matrix, consisting of $\mathbf{F}^{h}, h=$ $0,1,2,3$. Here, $\mathbf{F}^{h}$ is the $h$ times of discrete Fourier transform (DFT) matrix. And 

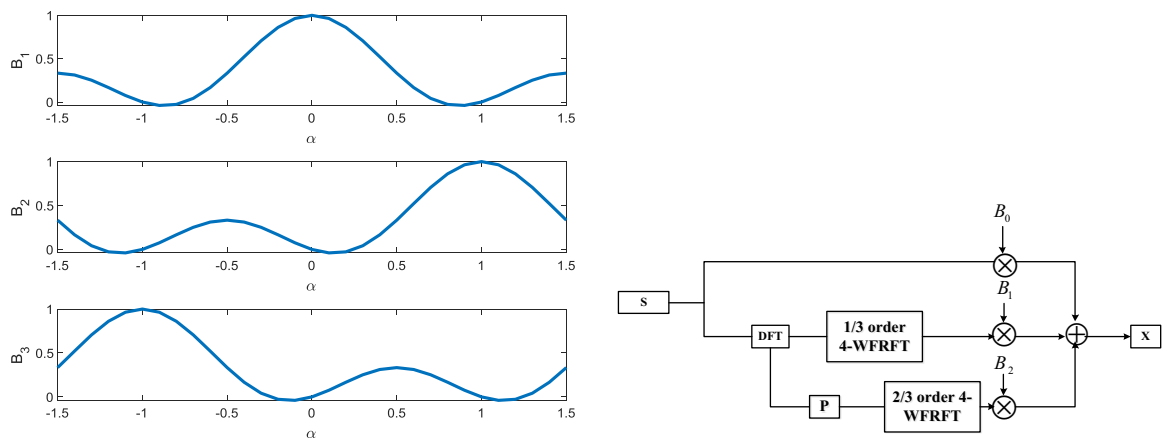

Figure 1. The amplitude of $B_{l}(\alpha)$.

Figure 2. The Implement of 3-WFRFT

$[\mathbf{F}]_{m, n}=e^{-j 2 \pi m n / N}$, where $m, n \in \mathbb{Z}, m, n \in[0, N-1]$. Specially, $\mathbf{F}^{h}$ will be degenerated to unit matrix if $l=0$, moreover $\mathbf{F}^{h}$ will become the DFT matrix $\mathbf{F}$ when $h=1$. The 4-WFRFT matrix, i.e., $\mathbf{W}_{4}^{\frac{4}{3} l}$, can be as:

$$
\mathbf{W}_{4}^{\frac{4}{3} l}=\sum_{h=0}^{3} A_{h}\left(\frac{4}{3} l\right) \mathbf{F}^{h}
$$

$A_{h}(\beta)(h \in \mathbb{Z}, h \in[0,3])$ is the 4 -WFRFT weighted coefficient, and can be described as:

$$
A_{h}(\beta)=\frac{1}{4} \frac{1-e^{-j 2 \pi(\beta-h)}}{1-e^{-j 2 \pi(\beta-h) / 4}}
$$

And $B_{l}(\alpha)$ is termed as the 3-WFRFT coefficient, and can be expressed as:

$$
B_{l}(\alpha)=\frac{1}{3} \sum_{n=0}^{2} \exp \left[-\frac{2 \pi j n(\alpha-l)}{3}\right], l=0,1,2 . \alpha \in[-1.5,1.5]
$$

The amplitude of $B_{l}(\alpha)$ can be described as Figure 1. From Figure 1, it can be observed that, when $\alpha=0$, only the $B_{0}=1$. The 3-WFRFT matrix will be degenerated to unit matrix.

\subsection{Property of $3-W F R F T$}

Property 1 For any $\alpha$ and $\beta$, the additive of 3-WFRFT holds:

$$
\mathbf{W}_{3}^{\alpha+\beta}=\mathbf{W}_{3}^{\alpha} \mathbf{W}_{3}^{\beta}=\mathbf{W}_{3}^{\beta} \mathbf{W}_{3}^{\alpha}
$$

Property 2 For any $\alpha$, the periodicity of 3-WFRFT holds:

$$
\mathbf{W}_{3}^{\alpha}=\mathbf{W}_{3}^{\alpha+3}
$$


Property 3 For any $\alpha$, the reversibility of 3-WFRFT holds:

$$
\mathbf{W}_{3}^{\alpha} \mathbf{W}_{3}^{-\alpha}=\mathbf{W}_{3}^{\alpha}\left(\mathbf{W}_{3}^{\alpha}\right)^{T}=\mathbf{I}
$$

where (.) $)^{T}$ is the transpose of matrix, and $\mathbf{I}$ is the unit matrix.

The proof processing of three properties is straight and omitted here. Specially, 3WFRFT matrix is the orthogonal one based upon the reversibility property, which is easy to be implemented in reality.

\section{The Implement of 3-WFRFT and Its PAPR Analysis}

\subsection{The Implement of 3-WFRFT}

The implement of 3-WFRFT, based 4-WFRFT, DFT and permutation matrix, is demonstrated in Figure.2. The 4-WFRFT operation has been provided in [15], DFT operation is based upon fast Fourier transform (FFT).

The implement of 3-WFRFT is simple. And also, we can find that the 3-WFRFT signal can be consisted by single carrier (SC), weighted type fractional Fourier transfor$\mathrm{m}$ domain signals. In fact, the 4-WFRFT is consisted of single carrier and orthogonal frequency division multiplexing (OFDM) signals. Thence, 3-WFRFT can also be constituted by SC and OFDM signal, since the 3-WFRFT is based upon 4-WFRFT in this paper.

Based upon the previous reasons, it interests us that what about the peak to average power ratio (PAPR) of 3-WFRFT, comparing with OFDM and SC. Thence, we propose the PAPR of 3-WFRFT based generalized hybrid carrier (GHC) in the next section.

\subsection{The PAPR of $G H C$}

The peak to average power ratio (PAPR) of GHC based with 3-WFRFT signal $X=$ $\left\{x_{0}, x_{1}, \ldots, x_{N-2}, x_{N-1}\right\}$ in a given frame is defined as follows:

$$
\mathbf{P A P R}_{X}=\frac{\max _{n \in[0, N-1]}\left|x_{n}\right|^{2}}{E\left|x_{n}\right|^{2}}
$$

PAPR, in general, can be considered as random variable and be given by the complementary cumulative density function ( CCDF)[3]. It can be defined the probability value that PAPR exceeds an preset value $\mathbf{P A P R} \mathbf{R}_{0}>0$.

$$
\mathbf{C C D F}=\operatorname{Pr}\left(\mathbf{P A P R}>\mathbf{P A P R}_{0}\right)
$$

Obviously, the PAPR of OFDM and SC can be also described as aforesaid. However, the structures of signal for GHC, OFDM and SC system are different. Thence, the PAPR level of them will be different. Based upon the above, we will take some numerical simulations to compare the PAPR of GHC, OFDM and SC signals in the next section. 


\section{Simulations and Discussions}

To verify the PAPR performance superiority of GHC system, in comparison to the SC and OFDM system, we present the numerical simulation under the additive white gaussian noise (AWGN) channels. Here, we set the testing signal are phase-shift keying (PSK) and quadrature amplitude modulation (QAM) signal. Without loss of generalization, we choose quadrature phase-shift keying (QPSK) and 16QAM as the numerical simulation signals.

We can observe that, from Figure 3, given that CCDF $=10^{-2}$, the PAPR of GHC based on 3-WFRFT with $\alpha=0.2$ is about $5 \mathrm{~dB}, \alpha=0.4$ is $2 \mathrm{~dB}$, and $\alpha=0.4$ is $1 \mathrm{~dB}$ superior over that of OFDM signal level with QPSK modulation. The PAPR of SC with QPSK modulation is nearly zero. With the $\alpha(\alpha>0.7)$ increasing, the PAPR of GHC with 3-WFRFT signal level is almost the same.

It can be demonstrated that, from the Figure 4, given that CCDF $=10^{-2}$, the PAPR of GHC based on 3-WFRFT with $\alpha=0.2$ is about $4 \mathrm{~dB}, \alpha=0.4$ is $2 \mathrm{~dB}$, and $\alpha=0.5$ is $0.5 \mathrm{~dB}$ superior over that of OFDM signal level with 16QAM modulation. The PAPR of SC with 16QAM modulation is nearly zero. The reason why PAPR of GHC is superior over OFDM maybe that, the GHC with 3-WFRFT will be consisted of SC and OFDM signals. When $\alpha$ is small than 0.5 , the SC signal components is higher than OFDM signals. Thence, the PAPR property is near to that of SC system. And also, the 3-WFRFT will be near to OFDM signal with the transform order $\alpha$ increasing.

The 3-WFRFT based GHC system proposed in this paper, can be applied into PAPR suppression, especially in the wireless communication systems with non-linear high non-linear power amplifier (HPA), where the proposed 3-WFRFT will improve the PAPR suppression performance.

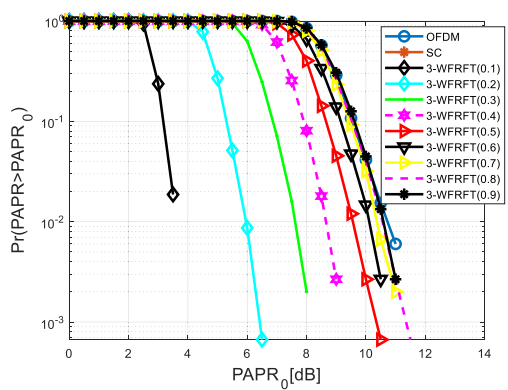

Figure 3. The PAPR of GHC, SCM and OFDM Figure 4. The PAPR of GHC ,SCM and OFDM systems with QPSK signals

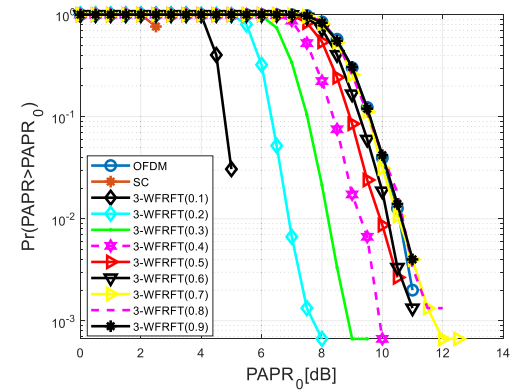

systems with 16QAM signals

\section{Conclusion}

In this paper, we proposed GHC system based upon 3-WFRFT to reduce the PAPR of communication system. The properties of 3-WFRFT has been explored in this paper. It can be demonstrated, from the numerical simulations, the PAPR of GHC system with 3-WFRFT is superior over OFDM system. In the future, we will explore 3-WFRFT with more applications over wireless communication. 


\section{Acknowledgements}

The work is supported by the fund of the National Key Research and Development Program of China (under grant 254). And this work is also supported by Science and Technology on Communication Networks Laboratory.

\section{References}

[1] T. Jiang and Y. Wu: An overview: Peak-to-Average power ratio reduction techniques for OFDM signals, IEEE Trans. Broadcast. 2008 54(2): 257-268.

[2] S. B. Weinstein: The history of orthogonal frequency-division multiplexing [history of communications],IEEE Communications Magazine. 2009 47(11): 26-35.

[3] D.D. Prasad and M.V Nageswara Rao: A Hybrid Companding Transform Technique for PAPR Reduction of OFDM Signals, International Conference on Electromagnetic Interference and Compatibility, 2015 1, 263-268.

[4] H. Joo: New PTS Schemes for PAPR Reduction of OFDM Signals Without Side Information, IEEE Transaction on Broadcasting, 201763 (3), 562-570.

[5] H. Hu, L. Zhang, X. Chen and Z. Wu: Recursive Carrier Interferometry Aided High Data Rate OFDM Systems With PAPR Suppression, Phase Noise Rejection, and Carrier Frequency Offsets Compensation, IEEE Transactions on Vehicular Technology, 201968 (4), 3655-3671.

[6] W. Jiang, X. Kuai, X. Yuan, W. Liu and Z. Song: Sparsity-Learning-Based Iterative Compensation for Filtered-OFDM With Clipping, IEEE Communications Letters, 202024 (11), 2483-2487.

[7] X. Cheng, D. Liu, W. Shi, Y. Zhao and D. Kong: A Novel Conversion Vector-based Low-Complexity SLM Scheme for PAPR Reduction in FBMC/OQAM Systems., IEEE Transaction on Broadcasting, 202066 (3), 656-666.

[8] Q. Ran, D. S. Yeung, C. C. Eric Tsang and et.al: General Multifractional Fourier Transform Method Based on the Generalized Permutation Matrix Group, IEEE Trans. Signal Process., 2005, 53 (1), 83-98.

[9] H. M. Ozaktas, O. Arikan, A. Kutay and G. Bozdagi: Digital computation of the fractional Fourier transform, IEEE Trans. Signal Process., 1996, 44 (9), 2141-2150.

[10] G. Cariolaro, T. Erseghe, P. Kraniauskas and N. Laurenti: A unified framework for the fractional Fourier transforms, IEEE Trans. Signal Process., 1998, 46 (12), 32063219.

[11] G. Cariolaro, T. Erseghe, P. Kraniauskas and N. Laurenti: Multiplicity of Fractional Fourier Transform and Their Relationship, IEEE Trans. Signal Process., 2000, 48 (1), 227-241.

[12] T. Erseghe, P. Kraniauskas and G. Cariolaro: Unified fractional Fourier transform and sampling theorem, IEEE Trans. Signal Process., 1999, 47 (12), 3419-3423.

[13] S. C. Pei, M. H. Yeh and C. C. Tseng: Discrete fractional Fourier transform based on orthogonal projections, IEEE Trans. Signal Process., 1999, 47 (5), 1335-1348.

[14] Y. Li, Z. Song and X. Sha: The Multi-Weighted type Fractional Fourier Transform Scheme and Its Application over Wireless Communications, EURASIP Journal on wireless communications and networking, 2018 41: 1-10.

[15] Y. Li, X. Sha and K. Wang: Low Complexity Equalization of HCM Systems with DPFFT Demodulation over Doubly-Selective Channels, IEEE Signal Process. Lett.,2014 21 (7), 862-865. 